\title{
Energy of stratified magnetic fluid on a porous basis in the environment
}

\author{
Elvira Egereva $^{1, *}$, Artem Egerev $^{2}$ \\ ${ }^{1}$ Moscow State University of Civil Engineering, Yaroslavskoe shosse, 26, Moscow, 129337, Russia \\ ${ }^{2}$ National Research University Higher School of Economics, Myasnitskaya St. 20, Moscow, 101000, \\ Russia
}

\begin{abstract}
Stratified magnetic fluid on a porous basis leads to energy savings of the human economy associated with the environment, which goes beyond the usual biospheric transformations.
\end{abstract}

\section{Introduction}

The environment is penetrated with magnetic energy due to the Earth, which has a magnetic field. A device with a two-layer magnetic fluid on a porous basis helps save energy related to the environment. So, cooling lubricants with a magnetic fluid serve as an excellent pressure sealer in seals. Like the coil, inside of which there is a magnetic fluid that converts the energy of the oscillatory motion into electrical.

In mechanical engineering, for example, magnetic fluids are used as a lubricant having properties controlled by a magnetic field. To study the operation of bearings on magnetic lubricant, it is necessary to investigate the magnetic waves arising in this case, since they affect the movement of the liquid in a porous medium. Quite often, a variety of liquids are used in technology to transfer power or energy. For example, the bucket of a small excavator is driven by the pressure of the oil entering the hydraulic cylinders. You can also adjust the flow of liquid in the pipeline pre-installed on a given section of the pipe electromagnet and entering a small amount of magnetic fluid.

Magnetic fluid based on engine oils or cooling lubricants serves as an excellent sealer in various seals, bearings and complex assemblies of machines. Also, magnetic fluids are used in the technology of studying the interaction of Saccharomyces cerevisiae brewing yeast cells [1]. The problem of propagation of surface waves in a layer of magnetic fluid on a porous basis is considered in [5].

\section{Materials and methods}

The propagation of gravitational waves in a non-conductive magnetizing two-layer liquid located on a solid impermeable base is considered.

The coordinate system is chosen so that the $\mathrm{z}$ axis is directed vertically upwards, the plane $\mathrm{z}=0$ coincides with the surface of the section of the lower layer of the stratified

\footnotetext{
*Corresponding author: egerevaen@mail.ru
} 
liquid - an impermeable wall. Further, all values relating to the lower and upper layers of the magnetic fluid with different densities $(\rho 1>\rho 2)$ are denoted by numbers 1,2 , respectively (Fig. 1). The values relating to the atmosphere are indicated by a low index of 3.

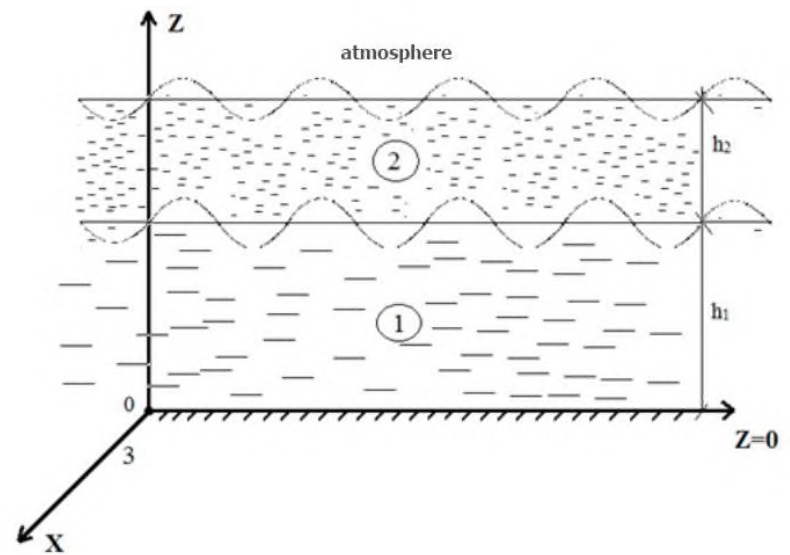

Fig. 1. Gravitational waves in a non-conductive magnetizing two-layer liquid located on a solid impermeable base.

The equations of motion of the magnetizing liquid at a constant magnetic permeability in the i-th layer $(i=1,2)$ have the form [7]:

$$
\begin{gathered}
\rho_{i} \frac{\partial \overrightarrow{u_{l}}}{\partial t}=-\nabla p_{i}+\rho_{i} \vec{g} \quad(\mathrm{i}=1,2), \\
\operatorname{div} \overrightarrow{u_{\imath}}=0 \quad(\mathrm{i}=1,2)
\end{gathered}
$$

In the layers of the lower and upper magnetic fluids, velocity potentials $\varphi_{1}, \varphi_{2}\left(\overrightarrow{u_{1}}=\right.$ $\left.\nabla \varphi_{1}(x, y, z, t), \overrightarrow{u_{2}}=\nabla \varphi_{2}(x, y, z, t)\right)$ satisfy the Laplace equations $\Delta \varphi_{i}=0 \quad(\mathrm{i}=1,2)$. The magnetic field potential in the $i$-th region $(i=1,2)$ is found from the equations:

$$
\Delta \Psi_{i}=0 \quad(\mathrm{i}=1,2),
$$

so far as $\operatorname{rot} \overrightarrow{H_{l}}=0$ and $\operatorname{div} \overrightarrow{H_{l}}=0, \overrightarrow{H_{l}}=\Delta \Psi_{i} \quad(\mathrm{i}=1,2)$.

The equation for the scalar potential in region 3 has the form:

$$
\Delta \Psi_{3}=0,
$$

The magnetic permeabilities $\mu_{1}, \mu_{2}, \mu_{3}$ in regions $1,2,3$ are further assumed to be constant.

Unperturbed magnetic fields in the absence of waves in the regions 1,2,3 are designated $\overrightarrow{H_{01}}, \overrightarrow{H_{02}}, \overrightarrow{H_{03}}$, respectively, where $\overrightarrow{H_{0 l}}-$ const $(i=1,2,3)$. When a wave appears, the magnetic field changes and should be written in the form: $\overrightarrow{H_{l}}=\overrightarrow{H_{0 l}}+\overrightarrow{H_{l}^{\prime}}$, where $\overrightarrow{H_{l}^{\prime}}$ small perturbations $(\mathrm{i}=1,2,3) \cdot \operatorname{rot} \overrightarrow{H_{0 l}}=0 ; \operatorname{rot} \overrightarrow{H_{0 l}^{\prime}}=0 ; \operatorname{rot} \overrightarrow{H_{l}^{\prime}}=0$ - equations for the perturbed magnetic field $(i=1,2,3)$. In addition, the equations $\operatorname{div} \overrightarrow{H_{0 l}}=0 ; \operatorname{div} \overrightarrow{H_{l}^{\prime}}=0(i=1,2,3)$ must be satisfied.

The potential of the perturbed magnetic field has the form:

$$
\Psi_{i}=\Psi_{0 i}+\Psi_{i}^{\prime} \quad(\mathrm{i}=1,2,3),
$$


Since curl $\operatorname{rot} \overrightarrow{H_{0 l}}=0 \quad(\mathrm{i}=1,2,3)$, then $\overrightarrow{H_{0 l}}=\nabla \Psi_{0 i}(\mathrm{i}=1,2,3)$. Therefore, $\Psi_{0 i}$ can be written in the form:

$$
\Psi_{0 i}=\overrightarrow{H_{0 i}} \cdot \vec{r}=H_{0 i x} \cdot x+H_{0 i y} \cdot y+H_{0 i z} \cdot z \quad(\mathrm{i}=1,2,3),
$$

where $H_{0 i x}=\frac{\partial \Psi_{0 i}}{\partial x} ; H_{0 i y}=\frac{\partial \Psi_{0 i}}{\partial y} ; H_{0 i z}=\frac{\partial \Psi_{0 i}}{\partial z}$.

The force in a magnetized medium has the form $[3,10]$ :

$$
\overrightarrow{f_{m}}=\frac{1}{4 \pi} \nabla \int_{0}^{H}\left[\mu-\rho\left(\frac{\partial H}{\partial \rho}\right)_{H, T}\right] H d H+\frac{\mu}{8 \pi} \nabla H^{2},
$$

Where $\mathrm{H}=|\vec{H}|, \mu=\mu(\rho, T, H)-$ is the magnetic permeability of the magnetic field, $\mathrm{H}$ is the magnetic field strength, $\rho$ - is the density, and T - is the temperature.

Further it is assumed that $\rho=$ const, $\mathrm{T}=$ const. We shall confine ourselves to the case of the initial section of the magnetization curve, when $\frac{\partial \mu}{\partial H} \neq 0$, without giving anything fundamentally new, only complicates the mathematical calculations. For $\mu=$ const, the force in the magnetized medium, calculated by formula (6), is equal to $\overrightarrow{\mathrm{f}_{\mathrm{m}}}=0$.

The boundary conditions on the interfaces are:

$$
\begin{gathered}
\mathrm{u}_{1 \mathrm{z}}=0 \quad(\mathrm{z}=0) ; \\
\Psi_{1}=0 \quad(\mathrm{z}=0) ; \\
u_{1 z} \frac{\partial \xi_{1}(x, y, t)}{\partial t} \quad\left(z=h_{1}+\xi_{1}(x, y, t)\right),
\end{gathered}
$$

where $\xi_{1}=\xi_{1}(x, y, t)$ - is the deviation of the disturbed surface of the lower layer of the magnetic fluid from the plane;

$$
\begin{array}{lll}
\text { 1) } & u_{1 z}=u_{2 z} & \left(\mathrm{z}=h_{1}+\xi_{1}(x, y, t)\right) \\
\text { 2) } & \Psi_{1}=\Psi_{2} \quad\left(\mathrm{z}=h_{1}+\xi_{1}(x, y, t)\right) \\
\text { 3) } & \mu_{1} \vec{n} \cdot \nabla \Psi_{1}= & \mu_{2} \vec{n} \cdot \nabla \Psi_{2} \quad\left(\mathrm{z}=h_{1}+\xi_{1}(x, y, t)\right)
\end{array}
$$

where $\vec{n}$ - is the normal vector to the interface of two layers of the magnetic fluid;

4) $p_{1}-\mu_{1} \frac{H_{1 n}^{2}}{4 \pi}+\frac{1}{4 \pi} \int_{0}^{H_{1}} \mu_{1} H d H-\left(p_{2}-\mu_{2} \frac{H_{2 n}^{2}}{4 \pi}+\frac{1}{4 \pi} \int_{0}^{H_{2}} \mu_{2} H d H\right)=-\alpha_{1} \Delta \xi_{1}(x, y, t)$ $\left(\mathrm{z}=h_{1}+\xi_{1}(x, y, t)\right)$

5) $u_{2 z} \frac{\partial \xi_{2}(x, y, t)}{\partial t} \quad\left(\mathrm{z}=h_{1}+h_{2}+\xi_{2}(x, y, t)\right)$,

where $\xi_{2}=\xi_{2}(x, y, t)$ - deviation of the perturbed surface of the upper layer of the stratified magnetic fluid from the plane [6];

6) $\Psi_{2}=\Psi_{3} \quad\left(\mathrm{z}=h_{1}+h_{2}+\xi_{2}(x, y, t)\right)$;

7) $\mu_{2} \vec{n} \cdot \nabla \Psi_{2}=\mu_{3} \vec{n} \cdot \nabla \Psi_{3} \quad\left(\mathrm{z}=h_{1}+h_{2}+\xi_{2}(x, y, t)\right)$,

where $\vec{n}$ - is the normal vector to the interface between the upper layer of the magnetic fluid and the atmosphere;

8) $p_{2}-\frac{\mu_{2}}{4 \pi} H_{2 n}^{2}+\frac{1}{4 \pi} \int_{0}^{H_{2}} \mu_{2} H d H-\left(p_{3}-\frac{\mu_{3}}{4 \pi} H_{3 n}^{2}+\frac{1}{4 \pi} \int_{0}^{H_{3}} \mu_{3} H d H\right)=-\alpha_{2} \Delta \xi_{2}(x, y, t)$ $\left(\mathrm{z}=h_{1}+h_{2}+\xi_{2}(x, y, t)\right)$;

9) $\Psi_{3}=0 \quad(z \rightarrow+\infty)$. 
At the interface of two layers of a magnetic fluid $\mathrm{z}=h_{1}+\xi_{1}(x, y, t)$, the normal vector $\vec{n}$ to the surface $f(x, y, z, t)=z-h_{1}-\xi_{1}(x, y, t)=0$ can be written in the form [2]:

$$
\vec{n}=\frac{\nabla f}{|\nabla f|}=\frac{\frac{\partial f}{\partial x} \overrightarrow{e_{1}}+\frac{\partial f}{\partial y} \overrightarrow{e_{2}}+\frac{\partial f}{\partial z} \overrightarrow{e_{3}}}{\sqrt{\left(\frac{\partial f}{\partial x}\right)^{2}+\left(\frac{\partial f}{\partial y}\right)^{2}+\left(\frac{\partial f}{\partial z}\right)^{2}}}=\frac{-\frac{\partial \xi_{2}}{\partial x} \overrightarrow{e_{1}}-\frac{\partial \xi_{2}}{\partial y} \overrightarrow{e_{2}}+\overrightarrow{e_{3}}}{\sqrt{\left(\frac{\partial \xi_{2}}{\partial x}\right)^{2}+\left(\frac{\partial \xi_{2}}{\partial y}\right)^{2}+1}} \approx-\frac{\partial \xi_{2}}{\partial x} \overrightarrow{e_{1}}-\frac{\partial \xi_{2}}{\partial y} \overrightarrow{e_{2}}+\overrightarrow{e_{3}},
$$

where $\overrightarrow{e_{1}}, \overrightarrow{e_{2}}, \overrightarrow{e_{3}}$ - are the basis vectors of the chosen coordinate system. In the following, it is assumed that $h_{1}$ and $h_{2}$ are constants.

Thus, considering the linear approximation at the interface of two layers of a stratified magnetic fluid, we have:

$$
n_{x}=-\frac{\partial \xi_{1}}{\partial x} ; \quad n_{y}=-\frac{\partial \xi_{1}}{\partial y} ; \quad n_{z}=1
$$

The seventh boundary condition takes into account:

$$
\begin{gathered}
\overrightarrow{H_{J}^{2}}=\left(\overrightarrow{H_{0 j}}+\overrightarrow{H_{j}^{\prime}}\right)^{2}=\overrightarrow{H_{0 j}^{2}}+2 \overrightarrow{H_{0 j}} \cdot \overrightarrow{H_{j}^{\prime}}+\overrightarrow{H_{j}^{\prime 2}}=H_{0 j x}^{2}+H_{0 j y}^{2}+H_{0 j z}^{2}+2 H_{0 j x} \frac{\partial \Psi_{j}^{\prime}}{\partial x}+ \\
2 H_{0 j y} \frac{\partial \psi_{j}^{\prime}}{\partial y}+2 H_{0 j z} \frac{\partial \Psi_{j}^{\prime}}{\partial z}+H_{j x}^{\prime 2}+H_{j y}^{\prime 2}+H_{j z}^{\prime 2}(\mathrm{j}=1,2,3) .
\end{gathered}
$$

Further we have:

$$
\begin{gathered}
H_{j n}=\overrightarrow{H_{j}} \cdot \vec{n}=H_{j x} \cdot n_{x}+H_{j y} \cdot n_{y}+H_{j z} \cdot n_{z}=\left(H_{0 j x}+H_{j x}^{\prime}\right) n_{x}+\left(H_{0 j y}+H_{j y}^{\prime}\right) n_{y}+ \\
\left(H_{0 j z}+H_{j z}^{\prime}\right) n_{z}=\left(H_{0 j x}+\frac{\partial \Psi_{j}}{\partial x}\right) n_{x}+\left(H_{0 j y}+\frac{\partial \Psi_{j}}{\partial y}\right) n_{y}+\left(H_{0 j z}+H_{j z}^{\prime}\right) n_{z}(\mathrm{j}=1,2,3) .
\end{gathered}
$$

In the seventh, eleventh boundary conditions for pressure, it is taken into account that the definite integral has the form:

$$
\begin{gathered}
\int_{0}^{H_{i}} \mu_{i} H_{i} d H_{i}=\frac{\mu_{i} H_{i}^{2}}{2}(i=1,2,3) ; \alpha_{1} \Delta \xi_{1}= \\
p_{1} \text {. }
\end{gathered}
$$

In the initial boundary conditions, it is necessary to take into account that for the unperturbed state, the boundary conditions have the form:

$$
\begin{aligned}
& \Psi_{10}=0 \quad(\mathrm{z}=0) ; \\
& \Psi_{10}=\Psi_{20} \quad(\mathrm{z}=0) ; \\
& \mu_{1} \frac{\partial \Psi_{10}}{\partial z}=\mu_{2} \frac{\partial \Psi_{20}}{\partial z} \quad\left(\mathrm{z}=\mathrm{h}_{1}\right) ; \\
& p_{10}-\frac{\mu_{1}}{4 \pi} H_{01 z}^{2}+\frac{\mu_{1}}{8 \pi} H_{01}^{2}=p_{20}-\frac{\mu_{2}}{4 \pi} H_{02 z}^{2}+\frac{\mu_{2}}{8 \pi} H_{02}^{2} \quad\left(\mathrm{z}=\mathrm{h}_{1}\right) ; \\
& \Psi_{20}=\Psi_{30} \quad\left(\mathrm{z}=\mathrm{h}_{1}+\mathrm{h}_{2}\right) ; \\
& \mu_{2} \frac{\partial \Psi_{20}}{\partial z}=\mu_{3} \frac{\partial \Psi_{30}}{\partial z} \quad\left(\mathrm{z}=\mathrm{h}_{1}+\mathrm{h}_{2}\right) ; \\
& p_{20}-\frac{\mu_{2}}{4 \pi} H_{02 z}^{2}+\frac{\mu_{2}}{8 \pi} H_{02}^{2}=p_{30}-\frac{\mu_{3}}{4 \pi} H_{03 z}^{2}+\frac{\mu_{3}}{8 \pi} H_{03}^{2} \quad\left(\mathrm{z}=\mathrm{h}_{1}+\mathrm{h}_{2}\right) ; \\
& \Psi_{30}=0 \quad(z \rightarrow+\infty) .
\end{aligned}
$$

The solution of the initial equations with boundary conditions is sought in the form of damped traveling waves:

$$
\varphi_{j}(x, y, z, t)=\Phi_{j}(z) \exp \left[i\left(-\omega \mathrm{t}+\mathrm{k}_{1} \mathrm{x}+\mathrm{k}_{2} \mathrm{y}\right)\right] \quad(\mathrm{j}=1,2)
$$




$$
\Psi_{k}^{\prime}(x, y, z, t)=\Psi_{k}(z) \exp \left[i\left(-\omega \mathrm{t}+\mathrm{k}_{1} \mathrm{x}+\mathrm{k}_{2} \mathrm{y}\right)\right] \quad(\mathrm{k}=1,2,3),
$$

where $\omega$ - is the vibration frequency of the wave, $k_{1}, k_{2}$ are real wave numbers characterizing wave propagation along the $\mathrm{x}$, $\mathrm{y}$ axis, respectively.

$$
k^{2}=k_{1}^{2}+k_{2}^{2} \quad\left(k=\frac{2 \pi}{\lambda}, \lambda \text {-wavelength }\right) .
$$

Substituting the expressions (9) in the initial equations (1) - (3), we obtain homogeneous ordinary differential equations of the second order with respect to z:

$$
\Phi_{i}^{\prime \prime}(z)-k^{2} \Phi_{i}(z)=0 ; \quad(\mathrm{i}=1,2) ; \Psi_{j}^{\prime \prime}(z)-k^{2} \Psi_{j}(z)=0 \quad(\mathrm{j}=1,2,3),
$$

Here the prime denotes differentiation with respect to $\mathrm{z}$.

Solutions of the system of differential equations (10) are functions of the form:

$$
\begin{gathered}
\Phi_{1}(z)=C_{1} \exp \left(-k_{z}\right)+C_{2} \exp \left(k_{z}\right) ; \Phi_{2}(z)=C_{3} \exp \left(-k_{z}\right)+C_{4} \exp \left(k_{z}\right) ; \\
\Psi_{1}(z)=C_{5} \exp \left(-k_{z}\right)+C_{6} \exp \left(k_{z}\right) ; \Psi_{2}(z)=C_{7} \exp \left(-k_{z}\right)+C_{8} \exp \left(k_{z}\right) ; \\
\Psi_{3}(z)=C_{9} \exp \left(-k_{z}\right)+C_{10} \exp \left(k_{z}\right),
\end{gathered}
$$

where $\mathrm{C}_{1}-\mathrm{C}_{10}$ - are integration constants.

We substitute the expression for the amplitudes $\Phi_{\mathrm{i}}(\mathrm{z}) ; \Psi_{j}$ to the boundary conditions (7), we obtain a linear system of ten equations with ten unknowns $\mathrm{C}_{1}-\mathrm{C}_{10}$ :

$$
\begin{gathered}
C_{1}+C_{2}=0 \\
C_{5}+C_{6}=0 ; \\
-C_{1}+C_{2} e^{2 k h_{1}}+C_{3}-C_{4} e^{2 k h_{1}}=0 ; \\
-k\left(H_{01 z}-H_{02 z}\right) C_{1}+k\left(H_{01 z}-H_{02 z}\right) e^{2 k h_{1}} C_{2}-i \omega C_{5}-i \omega e^{2 k h_{1}} C_{6}+i \omega C_{7}+i \omega e^{2 k h_{1}} C_{8} \\
=0 ; \\
-\mu_{1} C_{5}+\mu_{1} e^{2 k h_{1}} C_{6}+\mu_{2} C_{7}-\mu_{2} e^{2 k h_{1}} C_{8}=0 ; \\
\left(\rho_{1}\left(-\frac{\omega^{2}}{g k}-1\right)+\rho_{2}-\frac{\alpha_{1} k^{2}}{g}\right) C_{1}+\left(\rho_{1}\left(-\frac{\omega^{2}}{g k}+1\right)-\rho_{2}+\frac{\alpha_{1} k^{2}}{g}\right) e^{2 k h_{1}} C_{2}+\rho_{2} \frac{\omega^{2}}{g k} C_{3}+ \\
\rho_{2} \frac{\omega^{2}}{g k} C_{4} e^{2 k h_{1}}+i \omega \frac{\mu_{1}}{4 \pi g k}\left(i \vec{k} \cdot \overrightarrow{H_{01}}+k H_{01 z}\right) C_{5}+i \omega \frac{\mu_{1}}{4 \pi g k}\left(i \vec{k} \cdot \overrightarrow{H_{01}}-k H_{01 z}\right) C_{6} e^{2 k h_{1}}- \\
i \omega \frac{\mu_{2}}{4 \pi g k}\left\{i \vec{k} \cdot \overrightarrow{H_{02}}+k H_{02 z}\right\} C_{7}-i \omega \frac{\mu_{2}}{4 \pi g k}\left\{i \vec{k} \cdot \overrightarrow{H_{02}}-k H_{02 z}\right\} C_{8} e^{2 k h_{1}}=0 ; \\
-k\left(H_{02 z}-H_{03 z}\right) C_{3}+k\left(H_{02 z}-H_{03 z}\right) e^{2 k\left(h_{1}+h_{2}\right)} C_{4}-i \omega C_{7}-i \omega e^{2 k\left(h_{1}+h_{2}\right)} C_{8}+i \omega C_{9} \\
+i \omega e^{2 k\left(h_{1}+h_{2}\right)} C_{10}=0 ; \\
-\mu_{2} C_{7}+\mu_{2} e^{2 k\left(h_{1}+h_{2}\right)} C_{8}+\mu_{3} C_{9}-\mu_{3} e^{2 k\left(h_{1}+h_{2}\right)} C_{10}=0 ; \\
\rho_{2}\left(\left(-\frac{\omega^{2}}{g k}-1\right)-\frac{\alpha_{2} k^{2}}{g}\right) C_{3}+\left(\rho_{3}\left(-\frac{\omega^{2}}{g k}+1\right)+\frac{\alpha_{2} k^{2}}{g}\right) e^{2 k\left(h_{1}+h_{2}\right)} C_{4}+i \omega \frac{\mu_{2}}{4 \pi g k}\left\{i \vec{k} \cdot \overrightarrow{H_{02}}+\right. \\
\left.k H_{02 z}\right\} C_{7}+i \omega \frac{\mu_{2}}{4 \pi g k}\left\{i \vec{k} \cdot \overrightarrow{H_{02}}-k H_{02 z}\right\} e^{2 k\left(h_{1}+h_{2}\right)} C_{8}-i \omega \frac{\mu_{3}}{4 \pi g k}\left\{i \vec{k} \cdot \overrightarrow{H_{03}}+k H_{03 z}\right\} C_{9}- \\
i \omega \frac{\mu_{3}}{4 \pi g k}\left\{i \vec{k} \cdot \overrightarrow{H_{03}}-k H_{03 z}\right\} e^{2 k\left(h_{1}+h_{2}\right)} C_{10}=0 ; \\
\hline
\end{gathered}
$$

Equating the determinant of the resulting system of equations to zero, we write the dispersion equation in the form:

$$
a \Omega^{2}+b \Omega+c=0
$$


where the following notation is introduced: $\Omega=\omega^{2}$,

$$
\begin{gathered}
a=\frac{\rho_{2}}{(g k)^{2}}\left\{\rho_{2}\left\langle\left(\mu_{2}-\mu_{3}\right)\left[-\mu_{1} a_{9}+\mu_{2} a_{10}\right]-\left(\mu_{2}+\mu_{3}\right)\left(\mu_{1} a_{7}+\mu_{2} a_{8}\right)\right\rangle+\rho_{1}\left\langle\left(\mu_{2}-\right.\right.\right. \\
\left.\left.\left.\mu_{3}\right)\left(-\mu_{1} a_{12}+\mu_{2} a_{14}\right)-\left(\mu_{2}+\mu_{3}\right)\left(\mu_{1} a_{11}+\mu_{2} a_{16}\right)\right\rangle\right\} \\
b=-\left\{\frac{\mu_{2} \mu_{3}}{4 \pi g^{2}}\left(H_{02 z}-H_{03 z}\right)^{2}\left[\rho_{2}\left(\mu_{1} a_{2}+\mu_{2} a_{3}\right)+\rho_{1}\left(\mu_{1} a_{4}+\mu_{2} a_{1}\right)\right]+\frac{\mu_{1} \mu_{2}}{4 \pi g^{2}}\left(H_{01 z}-\right.\right. \\
\left.H_{02 z}\right)^{2} \rho_{2}\left[\left(\mu_{2}+\mu_{3}\right) a_{16}-\left(\mu_{2}-\mu_{3}\right) a_{14}\right]+\frac{\rho_{2}}{g k}\left(\rho_{1}-\rho_{2}+\frac{\alpha_{1} k^{2}}{g}\right)\left\{-\left(\mu_{2}+\mu_{3}\right)\left(\mu_{1} a_{16}+\right.\right. \\
\left.\left.\mu_{2} a_{13}\right)+\left(\mu_{2}-\mu_{3}\right)\left(-\mu_{1} a_{14}+\mu_{2} a_{15}\right)\right\}+2 \rho_{2} \frac{\mu_{1} \mu_{2} \mu_{3}}{\pi g^{2}}\left(H_{01 z}-H_{02 z}\right)\left(H_{02 z}-H_{03 z}\right) a_{19}+ \\
\left(\rho_{2}+\frac{\alpha_{2} k^{2}}{g}\right) \frac{1}{g k}\left\{\rho_{2}\left[\left(\mu_{2}-\mu_{3}\right)\left(-\mu_{1} a_{14}+\mu_{2} a_{15}\right)-\left(\mu_{2}+\mu_{3}\right)\left(\mu_{1} a_{16}+\mu_{2} a_{13}\right)\right]+\right. \\
\left.\left.\rho_{1}\left[\left(\mu_{2}-\mu_{3}\right)\left(-\mu_{1} a_{17}+\mu_{2} a_{19}\right)-\left(\mu_{2}+\mu_{3}\right)\left(\mu_{1} a_{18}+\mu_{2} a_{17}\right)\right]\right\}\right\} ; \\
\left.\frac{\alpha_{1} k^{2}}{g}\right)\left(\mu_{1} a_{6}+\mu_{2} a_{5}\right)+\frac{\mu_{1} \mu_{2}}{4 \pi g} k\left(H_{01 z}-H_{02 z}\right)^{2}\left(\rho_{2}+\frac{\alpha_{2} k^{2}}{g}\right)\left\{\left(\mu_{2}+\mu_{3}\right) a_{7}-\left(\mu_{2}-\mu_{3}\right) a_{9}\right\}+ \\
\left(\rho_{2}+\frac{\alpha_{2} k^{2}}{g}\right)\left(\rho_{1}-\rho_{2}+\frac{\alpha_{1} k^{2}}{g}\right)\left(\left(\mu_{2}-\mu_{3}\right)\left(-\mu_{1} a_{9}+\mu_{2} a_{10}\right)-\left(\mu_{2}+\mu_{3}\right)\left(\mu_{1} a_{7}+\mu_{2} a_{8}\right)\right)
\end{gathered}
$$

We consider the case when $D \geq 0$, since there is no fluid for the equation $D<0$.

Thus, the frequency value is found from the dispersion equation by the formula:

$$
\omega^{2}=\frac{-b \pm \sqrt{D}}{2 a} .
$$

\section{Results of the study}

The results of the calculations are shown in the graphs of the dependence of the frequency of the wave oscillations on the wave number $\mathrm{k}$, the dimensionless quantities $x=\mathrm{h}_{2} / \mathrm{h}_{1}$ and $\sigma=\mathrm{h}_{2} / \lambda$, where $\lambda$ is the wavelength $(\lambda=2 \pi / \mathrm{k})$.

Figure 2 shows the curves calculated for the following values of the thickness of the lower layer of the magnetic fluid: $\mathrm{h}_{1}=20 ; 150 ; 300 ; 600 ; 1000 \mathrm{~cm}$, respectively. The thickness of the upper layer of the magnetic fluid is fixed $h_{2}=100 \mathrm{~cm}$. At the same time, the values characterizing the magnetic field have the following values: magnetic permeability in the corresponding liquid layer $\mu_{1}=1.25 ; \mu_{2}=1.5$; The value of the unperturbed magnetic field in the atmosphere is $\mathrm{H}_{03 \mathrm{z}}=300 \mathrm{Oe}$. 


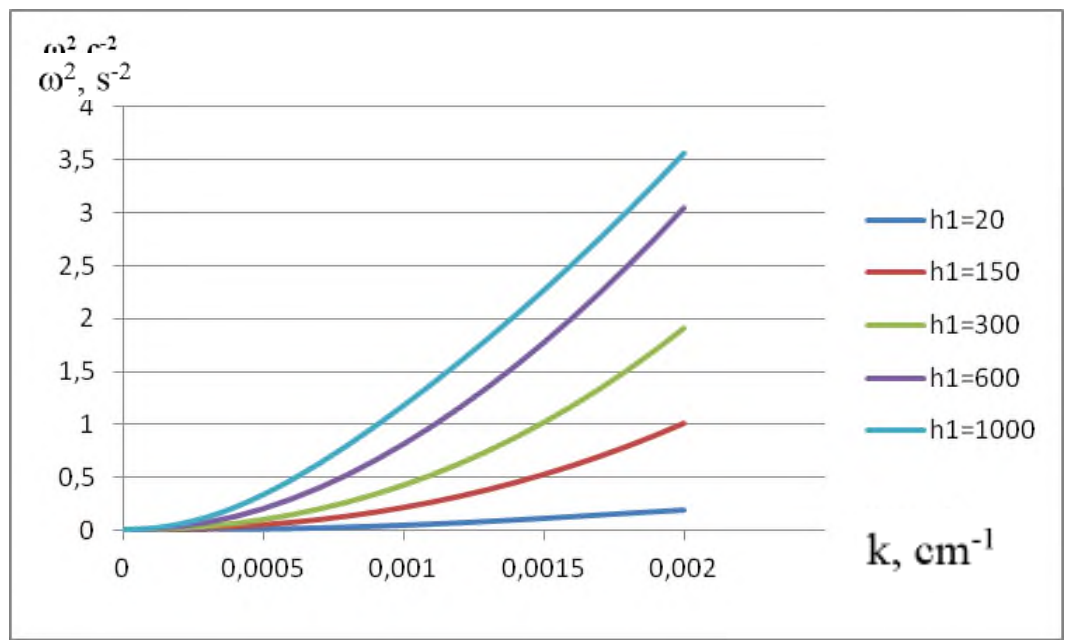

Fig. 2. Dependence of the frequency of the wave oscillations on the wave number k.

Figure 3 shows the dependence of the frequency of the wave oscillations on the dimensionless quantity $\sigma=\mathrm{h}_{2} / \lambda$ for a constant value of $\mathrm{h} 2=200 \mathrm{~cm}$ and $\mathrm{h} 1=100 ; 250 ; 500$; $750 ; 1000 \mathrm{~cm}$.

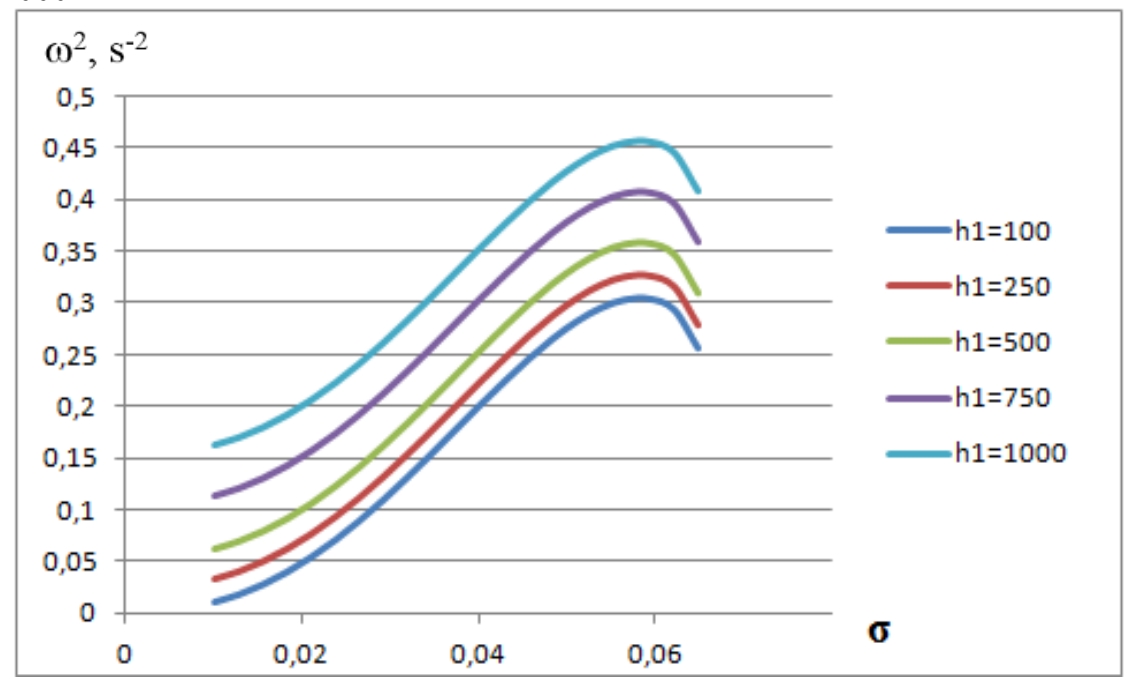

Fig. 3. The dependence of the frequency of the wave oscillations on the dimensionless quantity $\sigma=h_{2} / \lambda$ for a constant value of $h_{2}=200 \mathrm{~cm}$ and $h_{1}=100 ; 250 ; 500 ; 750 ; 1000 \mathrm{~cm}$.

Figure 4 depicts the dependence of the frequency of the wave oscillations on the dimensionless quantity $x=\mathrm{h}_{2} / \mathrm{h}_{1}$ at $\mathrm{k}=1 \cdot 10^{-3} ; 1,5 \cdot 10^{-3} ; 2 \cdot 10^{-3} \mathrm{~cm}^{-1}$. In this case, the values characterizing the magnetic field have the following values: the magnetic permeability in the corresponding liquid layer is $\mu_{1}=1.25 ; \mu_{2}=1.5$ and $\mu_{3}=1$. The value of the unperturbed magnetic field in the atmosphere is $\mathrm{H}_{03 \mathrm{z}}=300$ Oe. In Figure 4, the thickness of the lower layer of the magnetic fluid is $50 \mathrm{~cm}$ - fixed, and $\mathrm{h}_{2}=x \mathrm{~h}_{1}$. 


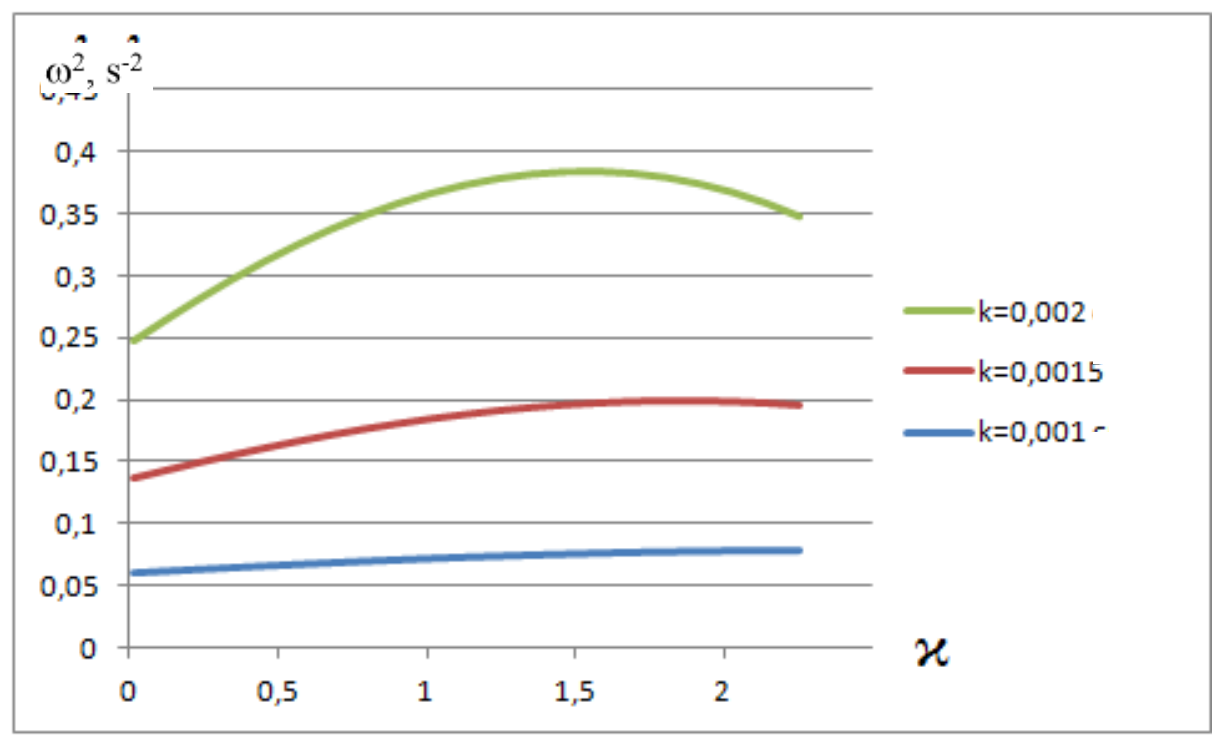

Fig. 4. The dependence of the frequency of the wave oscillations on the dimensionless quantity $x=\mathrm{h}_{2} / \mathrm{h}_{1}$ at $\mathrm{k}=1 \cdot 10^{-3} ; 1,5 \cdot 10^{-3} ; 2 \cdot 10^{-3} \mathrm{~cm}^{-1}$.

Figure 5 shows the frequency dependence of the wave number $k$ for different values of $\mu_{1}=1 ; 1.2 ; 1.4$. Calculations were carried out for a fixed thickness of the upper layer of the magnetic fluid $\mathrm{h}_{2}=100 \mathrm{~cm}, \mathrm{~h}_{1}=10 \mathrm{~cm}, \mathrm{H}_{03 \mathrm{z}}=100 \mathrm{Oe}, \mu_{2}=1.4$.

Figure 6 shows the dependence of the oscillation frequency of the wave on $\mathrm{H}_{03 \mathrm{z}}$, for different values of $h_{2}=100 ; 150 ; 200 \mathrm{~cm}$. In this case, $h_{1}=100 \mathrm{~cm} ; \mu_{1}=1,2 ; \mu_{2}=1.5$ and $\mu_{3}=1 ; \mathrm{k}=0.002 \mathrm{~cm}^{-1}$ - are fixed.

Figure 6 Captions should be typed in 9-point Times. They should be centred above the tables and flush left beneath the figures.

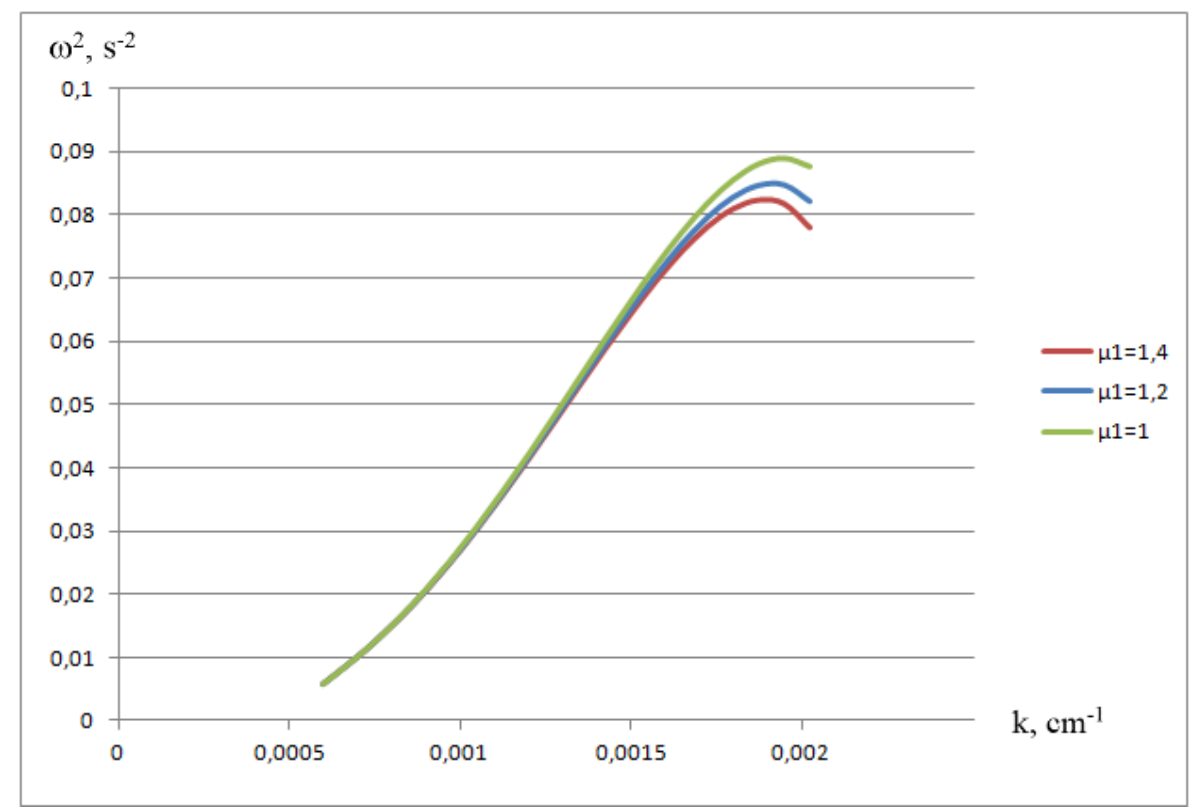

Fig. 5. Dependence of the wave number $\mathrm{k}$ for different values of $\mu_{1}=1 ; 1.2 ; 1.4$. 


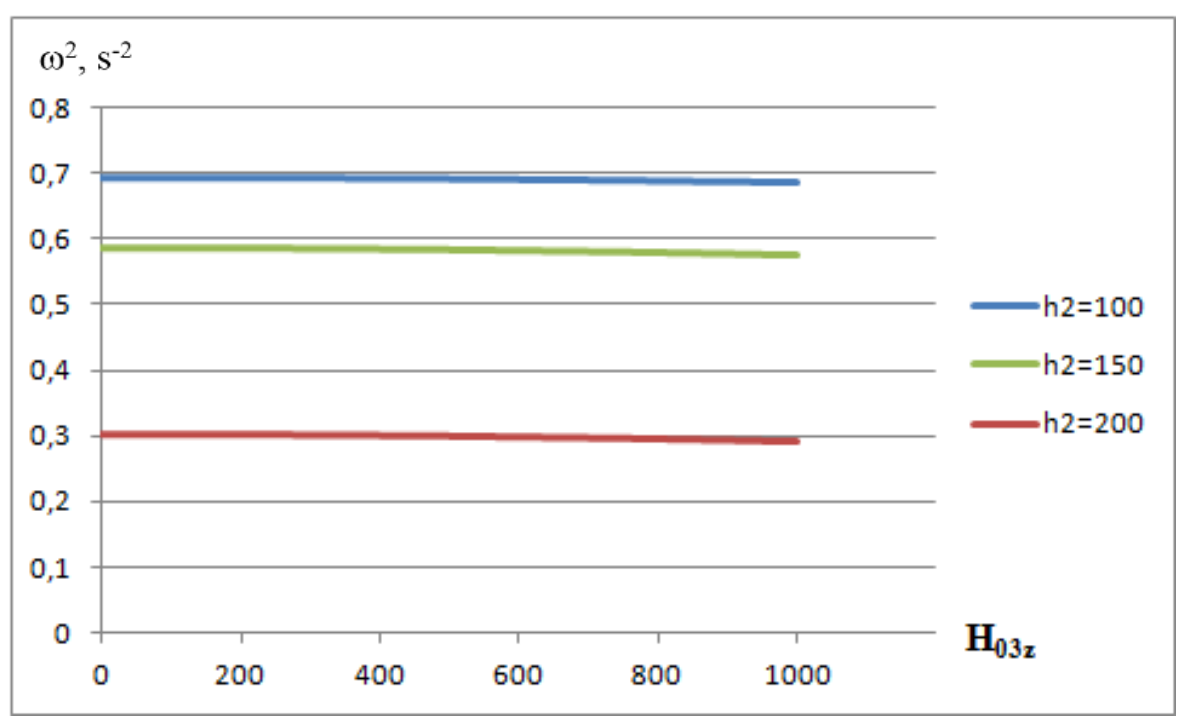

Fig. 6. Dependence of the oscillation frequency of the wave on $\mathrm{H}_{03 z}$, for different values of $\mathrm{h}_{2}=100$; $150 ; 200 \mathrm{~cm}$.

\section{Discussion and conclusions}

From the graphs shown in Figure 2 it follows that when the wave number increases, if the thickness of the lower layer of the magnetic fluid is fixed, the oscillation frequency increases. For a fixed value of the wave number and an increase in the thickness of the lower layer of the magnetic fluid, the frequency also increases, i.e. shorter waves decay faster than the longer ones.

From the graphs presented in Figure 3 it follows that for a fixed value of $h_{1}$ and an increase in $\sigma$, the frequency $\omega$ increases. If we fix $\sigma$ and increase the thickness of the lower liquid layer, then the frequency of the wave oscillations also increases. Thus, for fixed parameters characterizing a magnetic fluid, the dependence of the frequency $\omega$ on the dimensionless quantity $\sigma$ remains qualitatively the same as for the case when the magnetic field is absent [7].

Figure 4 shows that as the dimensionless quantity $x$ and the fixed wave number increase, the frequency increases. Conversely, for a fixed value of $x$ and an increase in the wave number $\mathrm{k}$, the slower the frequency decrease, the greater $\mathrm{k}$.

A decrease in the frequency for fixed values of the wave number $k$ and an increase in $\mu 1$ are shown in Figure 5. And also from this graph it follows that for a fixed value of $\mu 1$ and increasing the wave number $\mathrm{k}$, the frequency increases.

It can be seen in Figure 6 that the frequency depends weakly on $\mathrm{H} 03 \mathrm{z}$ for a fixed value of the thickness of the upper layer of the magnetic fluid. If we fix H03z, then the frequency $\omega$ decreases with increasing $\mathrm{h}_{2}$.

Thus, technological devices that use stratified magnetic fluid on a porous basis help supply energy to various devices, which help to save the energy of the environment. 


\section{References}

1. D.M. Aronbaev, S.D. Aronbaev, A.M. Nasimov, International Center for Science and Education 2(32), 32-36 (2017)

2. A.R. Baev, P.P. Prokhorenko, Engineering and Physics Journal, Institute for Heat and Mass Transfer 5(80), 133-140 (2007)

3. V.A. Barinov, N.G. Taktarov, Mathematical modeling of magnetodynamic surface waves: Textbook (Mordov Publishing House. Un-ta, Saransk, 1991)

4. F.P. Grosso, M.K. Bologa, Electronic processing of materials, Institute of Applied Physics of the Academy of Sciences of Moldova 6(260), 52-58 (2009)

5. E.N. Egereva, O.A. Runova, N.G. Taktarov, Izv. RAS. FGM 1, 153-162 (2015)

6. L.D. Landau, E.M. Lifshitz, Hydrodynamics (Nauka, Moscow, 1986)

7. V.B. Penkov, L.V. Levina, M.Yu. Levin, N.V. Kuzmenko, Science in Central Russia. All-Russian Scientific Research Institute for the Use of Machinery and Oil Products of the Russian Academy of Agricultural Sciences 2(20), 12-16 (2016)

8. V.M. Polunin, M.L. Boev, Min Tan Myo, L.I. Roslyakova, Izvestiya Yugo-Zapadnogo Gosudarstvennogo Universiteta. Series: Physics and Chemistry, South-Western state un. 1, 6-14 (2012)

9. N.G. Taktarov, Magnetic Hydrodynamics 4, 33-35 (1981)

10. A. Gedroics, H. Kalis, Magnetohydrodynamics 2(46), 153-170 (2010)

11. S. Hadgia, V. Iusan, A. Stanci, Journal of Magnetism and Magnetic Materials, Elsevier Science Publishing Company 1-3(201), 404-406 (1999)

12. G.M. Moatimid, Physica A: Statistical Mechanics and its Applications, Elsevier Science Publishing Company 3-4(328), 525-544 (2003) DOI: 10.1016/S03784371(03)00577-6 